\title{
INTERVENCIÓN EN LÍNEA PARA EL AUMENTO DE LA AUTOEFICACIA EN HABILIDADES DOCENTES POR INTERNET ANTE LA CONTINGENCIA DEL COVID-19
}

\section{Online Intervention for Self-efficacy Increase in Teaching's Abilities through the Internet at COVID-19 Contingency}

José Manuel SÁNCHEZ SORDO

Recibido: 30/06/2020; Aceptado: 16/09/2020; Publicado: 30/12/2020

Ref. Bibl. JOSÉ MANUEL SÁNCHEZ SORDO. Intervención en línea para el aumento de la autoeficacia en habilidades docentes por internet ante la contingencia del COVID-19. Enseñanza E Teaching, 38, 1-2020, 125-145.

RESUMEN: La contingencia del COVID-19 ha impactado en el sector educativo mexicano de manera significativa, implicando la transferencia de las actividades académicas presenciales al mundo digital. Lo cual para los docentes tradicionales posiblemente suponga un reto en función de sus creencias de autoeficacia para dichas actividades. Por lo cual el objetivo de este estudio fue evaluar los niveles de autoeficacia para las actividades docentes por internet $(n=70)$ e impactar en ellos por medio de la oferta de un curso de capacitación en línea sobre "Enseñanza asistida por computadora en la Era Digital». Los resultados muestran diferencias significativas en las puntuaciones de autoeficacia de los docentes antes y después del curso ( $\mathrm{p}<$ 0.05) con la prueba de rangos con signo de Wilcoxon.

Palabras clave: e-learning; COVID-19; autoeficacia; docencia; capacitación.

SUMMARY: The COVID-19 contingency has had a significant impact on the Mexican education sector, involving the transfer of face-to-face academic activities to the digital world, which for traditional teachers may possibly be a dare because of their self-efficacy beliefs for those activities. The objective of this work was to evaluate 

POR INTERNET ANTE LA CONTINGENCIA DEL COVID-19

the levels of self-efficacy for online teaching activities $(n=70)$ and to impact them through the offer of an online training course about "Computer Aided Teaching in the Digital Age». Results show significant differences in the levels of self-efficacy of the teachers before and after the course $(\mathrm{p}<0.05)$ with the Wilcoxon signed rank test.

Key words: e-learning; Covid-19; self-efficacy; teaching; training.

\section{INTRODUCCIÓN}

El surgimiento y desarrollo del Covid-19 ha obligado a los gobiernos del mundo a tomar medidas restrictivas y de confinamiento con la finalidad de evitar la propagación del virus; causando estragos a nivel mundial en la industria, el sector salud y el sector educativo entre otros como menciona Casanova (2020). Siendo el educativo uno de los sectores más afectados, pues miles de personas dejaron de recibir formación académica de manera presencial. Si bien esto implicó una problemática, en países con alto grado de desarrollo se pudo controlar, pues desde hace tiempo y de manera constante se implementan modalidades educativas a distancia, haciendo uso de herramientas tecnológicas accesibles la mayoría de la población.

Mas por su parte, en algunos países, la implementación de esta estrategia no es del todo viable. En México, como lo menciona Lloyd (2020), desde finales del siglo xx tras la aparición de internet se comenzó a hacer más visible la necesidad de implementar de forma cotidiana la tecnología con fines educativos; por lo que se iniciaron programas que hacían uso de las tecnologías de la información y comunicación (TIC) a nivel básico en las escuelas, mas desafortunadamente no han tenido gran impacto en el ámbito operativo y pedagógico.

Como consecuencia de esta implementación un tanto deficiente, en la actualidad, tras esta emergencia sanitaria, en México el panorama se ha visto complicado pues se intenta brindar educación a 36 millones de niños y adultos mediante el uso de las tecnologías, sin embargo, muchas personas se encuentran excluidas del aprovechamiento de las TIC (Lloyd, 2020). En este sentido el Gobierno Federal mexicano adoptó estrategias y acciones propuestas por la OMS y la UNESCO para enfrentar la contingencia, como lo es la puesta en marcha del programa de educación a distancia "Aprende en casa" a través de televisión e internet (Amador, 2020).

Tras la suspensión de labores presenciales en diferentes instituciones, Ruiz (2020) menciona que la Universidad Nacional Autónoma de México (UNAM) suspendió sus actividades presenciales el 23 de marzo bajo el lema "La UNAM no se detiene» pues las actividades continuaron, pero ahora bajo una modalidad en línea y a distancia. Consecuente a la implementación de esta medida, la universidad puso a disposición de sus docentes y alumnos herramientas tecnológicas y recursos digitales, para llevar a cabo las clases a distancia.

Si bien es cierto que se brindó una gran gama de herramientas tecnológicas, existe aún una brecha de acceso a la educación por medio de la tecnología, en parte por la deficiencia que padecen varios hogares mexicanos debido a la 
marginación, y por no contar con las competencias en el uso y aprovechamiento de las Tic. Lo cual hace referencia al poco desarrollo de las competencias para el uso y aprovechamiento de las tecnologías y los medios de comunicación tanto de estudiantes como de docentes, consecuencia de la lenta incorporación de estas tecnologías al sistema educativo mexicano. Una de las causas principales es la insuficiente y mal entendida formación del desarrollo didáctico de los profesores con relación a la creación de ambientes digitales de aprendizaje que no logran asimilarse como parte de una pedagogía digital (Lloyd, 2020; Sánchez, 2019). Para ello se considera necesario retomar en la formación docente los principios básicos de la alfabetización digital que contemplan el uso de tecnología, la comprensión crítica de la misma, así como la creación y comunicación de contenido digital en una gran diversidad de formatos (Arrieta y Montes, 2011).

En ese sentido las instituciones tienen un papel primordial en la formación de profesores dentro del campo de las Tic, valorando los diferentes factores relacionados con la predisposición del docente al momento de incorporar la tecnología en su quehacer, los cuales para Martínez et al. (2006) en López y Chávez (2013) son la experiencia con la que cuente en el uso de las computadoras, las actitudes y la autoeficacia. En este momento histórico que atraviesa el mundo, autores como Ferrari (2012), citado en Guimarães, Tejada y Pozos (2019), mencionan que la competencia digital es indispensable para un docente. Por ello, es importante que los docentes cuenten con diversas habilidades para poder realizar de manera exitosa su labor, como son: a) conocimiento de la materia que imparte, uso específico de las TIC en su campo de conocimiento; b) competencias pedagógicas, didáctica digital y tutoría; c) conocimiento de nuevos lenguajes tecnológicos y audiovisuales (Moreno y Gonzales, 2013).

Y si bien a pesar de que la mayoría de los profesores reconocen la importancia que tienen las Tic en la educación, no parecen integrarlas del todo, pues, las utilizan únicamente como una herramienta de consulta y comunicación (Arrieta y Montes, 2011). Siendo entonces el equipamiento y la preparación en herramientas digitales de los docentes un área de oportunidad para los directivos, pues independientemente de la brecha generacional, la formación de los profesores en materia tecnológica debe ser valorada desde el propio sistema educativo.

En la situación actual para el caso de los docentes mexicanos, Ruiz (2020) menciona que no hubo una capacitación previa a la situación de cuarentena ante el uso y manejo de los métodos digitales, lo cual los llevó a experimentar procesos de adaptación y aprendizaje en el aislamiento social que se vive actualmente. Siendo la improvisación a manera de autoalfabetización digital importante y necesaria durante esta etapa de cuarentena, pues esta preparación por parte de los profesores fue más allá de un aprendizaje casual, ya que implicó compromiso profesional a diferencia del acercamiento a las TIC para el entretenimiento. El autoaprendizaje improvisado requirió reconocer las debilidades en su uso, aprender a utilizarlas y aplicarlas con fines educativos (Díaz, 2020). Y si bien es cierto que el e-learning desde sus inicios en los años 90 ha sido una herramienta que facilita el aprendizaje; 

POR INTERNET ANTE LA CONTINGENCIA DEL COVID-19

en aquellos profesores que incursionan por primera vez en esta modalidad es una herramienta inútil, pues se busca hacer una réplica del salón de clases en un aula virtual.

La escuela presencial no puede ser sustituida de forma automática por la escuela a distancia. Cada una tiene características y funcionamientos distintos (Suárez y Anaya, 2004). En la educación tradicional los roles son marcados y verticales, sin embargo, en la modalidad en línea existe una redefinición de los roles entre el alumno y el profesor, ya que el uso de recursos electrónicos modifica y transforma el modo de relación entre el alumno y el profesor, así como entre los propios alumnos (Ruiz, 2020).

Es así que la educación a distancia replantea las funciones del docente, pues, en un ambiente tradicional de educación, la labor de un docente es intervenir en el proceso de enseñanza-aprendizaje mediante la interacción que se da en un salón de clases; sin embargo, en la labor de un docente desde una modalidad a distancia, según Maya (2019), las funciones de este cambian a asesoría, facilitación o tutoría, propiciando situaciones de aprendizaje y apoyo del desarrollo autónomo de cada alumno, situación retomada por las principales teorías del aprendizaje aplicadas a la Era Digital (Pons, 2013) como lo es el conectivismo, que plantea la autonomía del estudiante en la generación de sus propias redes personales de aprendizaje (Siemens, 2006; Downes, 2012).

En este sentido, algunas de las características del docente en línea para Maya (2019) son, por ejemplo, las contadas ocasiones en que coincidirá sincrónicamente con el estudiante. De igual modo los materiales audiovisuales son las fuentes principales de información de esta modalidad en la cual se encarga de atender al alumno cuando lo solicite y, solo en caso de que necesite ayuda, se le brindará, avanzando así al ritmo del estudiante con apertura de horario y opciones digitales de atención.

Dichos cambios en la actividad docente, así como la brecha digital derivada del desconocimiento o poco aprovechamiento de los profesores y del sistema educativo de las tecnologías digitales, impacta de manera directa en las creencias que los docentes tradicionales tienen sobre sí mismos y sus capacidades para ofertar formación a distancia y por internet ante situaciones emergentes como la actual contingencia del Covid-19. Por lo cual, evaluar la autoeficacia de los docentes como actores importantes de la dinámica de enseñanza por internet se torna relevante y necesario, pues muchos de ellos posiblemente no se consideran suficientemente capaces de dar frente al reto que implica la imprevista y "forzada" digitalización de sus funciones. En esta línea, el estudio de la autoeficacia (Bandura, 2010) puede ser definido desde el enfoque social-cognitivo como la función de los juicios o creencias que las personas tenemos sobre nosotros mismos con relación a la ejecución o desarrollo de ciertas conductas o tareas que implican determinado nivel de desafío, dichas creencias a su vez impactan en el éxito o fracaso del individuo al momento de llevar a cabo la acción. Por lo cual, el estudio de las creencias de autoeficacia se muestra como un elemento clave en la modificación de la conducta desde 
INTERVENCIÓN EN LÍNEA PARA EL AUMENTO DE LA AUTOEFICACIA EN HABILIDADES DOCENTES POR INTERNET ANTE LA CONTINGENCIA DEL COVID-19

este enfoque, ya que dichas creencias son producto de cuatro fuentes que al ser manipuladas pueden ejercer una influencia positiva en el individuo y su conducta. Dichas fuentes son, para Bandura (1997), los logros de ejecución, la experiencia vicaria, la persuasión y los estados emocionales.

Por lo cual en el marco de la situación actual del Covid-19, dado que lo más probable es que la normalidad que se conocía tarde en regresar, es tiempo de plantear una normalidad alternativa, en donde sea posible una alfabetización digital meramente pedagógica, la cual deberá apoyarse en las habilidades que los profesores adquirieron durante la cuarentena (Díaz, 2020). Planteando así para este estudio que, si se evalúan los niveles de autoeficacia para impartir clases por internet de los docentes que están atravesando la contingencia del COVID-19 y, posteriormente, se les enseña y capacita por medio de una intervención digital que incluya información y estrategias válidas y actuales sobre enseñanza y aprendizaje en línea, a nivel psicológico se estará interviniendo con sus logros de ejecución, pues los docentes estarán llevando a cabo ellos mismos de manera exitosa actividades de aprendizaje en línea, podrán de igual modo aprender de otros de forma vicaria (ya sea de sus compañeros o por medio de videos o tutoriales) y ambos elementos posiblemente conduzcan a estados emocionales que faciliten el aprendizaje de nuevas habilidades, lo cual en conjunto posibilitará que los niveles de creencia de autoeficacia en sus habilidades docentes por internet aumenten al igual que su desempeño. Aportando de este modo a la disminución de la brecha digital centrada en la educación y a la formación de docentes mejor preparados y con niveles considerables de autoeficacia para las nuevas necesidades que su quehacer profesional demanda, lo cual puede ser buen indicador para el éxito de futuras inclusiones tecnológicas en el ámbito educativo, como lo mencionan López y Chávez (2013).

\section{MÉTODO}

\subsection{Objetivo}

Ofertar un curso en línea de capacitación docente sobre "Enseñanza Asistida por Computadora en la Era Digital» para conocer su impacto en los niveles de autoeficacia para el desarrollo de actividades docentes a distancia por internet ante la contingencia del COVID-19.

\subsection{Tipo de estudio}

No experimental

\subsection{Alcance}

Descriptivo de diferencia de grupos. 

POR INTERNET ANTE LA CONTINGENCIA DEL COVID-19

\subsection{Hipótesis}

H0: Los niveles de autoeficacia para el desarrollo de actividades docentes en línea durante la contingencia del COVID-19 no aumentarán significativamente después de acreditar el curso en línea «Enseñanza Asistida por Computadora en la Era Digital".

H1: Los niveles de autoeficacia para el desarrollo de actividades docentes en línea durante la contingencia del Covid-19 aumentarán significativamente después de acreditar el curso en línea «Enseñanza Asistida por Computadora en la Era Digital».

\subsection{Muestra}

- No probabilística de 70 docentes de la UnAM; el $52 \%$ imparte clases a nivel universitario y el $48 \%$ en nivel medio superior.

- Sexo: 59 \% mujeres, $41 \%$ hombres.

- Edad promedio de los participantes: 45.8 años.

\subsection{Procedimiento}

Se comenzó por generar el espacio virtual donde se alojó el curso teóricopráctico «Enseñanza Asistida por Computadora en la Era Digital» con duración de 20 horas autoadministradas dentro un servidor Linux (CenTos 6) correspondiente al dominio web www.cognociendo.unam.mx.

El curso ofertado tuvo por objetivo mostrar a los docentes las nociones básicas de la enseñanza asistida por computadora y particularmente las teorías del aprendizaje y metodologías de enseñanza más acordes a la Era Digital de forma teórica y aplicada. A nivel instruccional se siguió un diseño con base en el conectivismo y los Entornos Personales de Aprendizaje (Sánchez, 2019; Siemens, 2006 y Downes 2012), para de este modo ir familiarizando a los participantes con dichas propuestas teórico-metodológicas al mismo tiempo que aprendían sobre los procesos de aprendizaje y enseñanza por internet de forma autorregulada.

En cuanto a contenidos y actividades, el curso consistió en las siguientes unidades:

Unidad 1. Enseñanza Asistida por Computadora.

Unidad 2. Teorías del aprendizaje y tecnología educativa.

Unidad 3. Hacia nuevos paradigmas: Conectivismo y conocimiento en Red.

Unidad 4. Práctica: desarrollo de un producto de aprendizaje.

Dentro de las unidades 1, 2 y 3 los participantes revisaron contenidos de tipo teórico y metodológico en múltiples formatos multimedia texto plano, imágenes, esquemas, videos y audio. En cada lección debían responder de manera acertada 
INTERVENCIÓN EN LÍNEA PARA EL AUMENTO DE LA AUTOEFICACIA EN HABILIDADES DOCENTES POR INTERNET ANTE LA CONTINGENCIA DEL COVID-19

a exámenes calificados de forma automática que les permitían avanzar al siguiente tema o unidad. De igual modo contaban con espacios para hacer anotaciones a manera de resumen sobre lo revisado, así como foros de discusión y una red social enlazada a Facebook para mantener comunicación entre sí y el personal del curso encargado de dar seguimiento. En el caso de la unidad 4 (Práctica: desarrollo de producto de aprendizaje), los participantes tuvieron que generar un producto digital de aprendizaje que abordara alguno de los temas revisados a lo largo de las primeras tres unidades, y para ello debían seleccionar un formato multimedia que les permitiera realizar el producto (video, infografía o presentación en Prezi). Si bien muchos de los participantes posiblemente desconocían el uso de las herramientas web requeridas para realizar dichas actividades, se incluyeron tutoriales en video así como la instrucción de que buscaran en la web recursos que les permitieran aprender cómo hacerlo, siguiendo así una de las premisas básicas del conectivismo, que plantea que el aprendizaje es el proceso autónomo por medio del cual generamos redes de información en internet que nos permiten extraer conocimiento (Siemens, 2004; Sánchez, 2019b). Como resultado de este diseño instruccional se esperaba que los docentes participantes mejoraran sus habilidades para el aprendizaje en línea al mismo tiempo que conocían nuevos métodos y herramientas para llevar a cabo sus demandas profesionales por medio de internet, lo cual en conjunto suponemos que aumentaría sus niveles de autoeficacia para la docencia en línea.

Posterior a la construcción del curso, se desarrolló con base en la literatura (Vanden, 2020; UnEsCO, 2020; Area y Adell, 2009; Maya, 2019; Adell y Sales, 1999) una escala de autoeficacia para las habilidades docentes por internet. El fin de la aplicación de esta escala fue obtener las medidas de autoeficacia de los participantes antes de iniciar el curso y al concluirlo (pretest-postest), para de este modo ver si la capacitación brindada tuvo o no impacto significativo en los participantes.

Para el desarrollo de la escala, se siguieron las propuestas de Bandura (2006) en la Guía para la construcción de escalas de Autoeficacia que, de manera general, plantea utilizar puntuaciones del 0 al 10 para los reactivos, centrarse en un constructo diferenciado de autoeficacia para una conducta o habilidad en concreto, así como no confundir la autoeficacia con autoestima o expectativa de resultados. A nivel métrico la escala desarrollada cuenta con un índice de validez de contenido (Lawshe, 1975; Tristán, 2008) de CVI $=.88$ y una consistencia interna por el método Split-half de Spearman-Brown (Silva, 2004) de $r=.98$, y se compone de 18 ítems que se enlistan a continuación:

Soy capaz de...

1. Realizar actividades de docencia exclusivamente por internet.

2. Aplicar los conocimientos teóricos sobre enseñanza que poseo a los entornos digitales de aprendizaje.

3. Sostener videoconferencias dinámicas con mis estudiantes que no excedan los 45 minutos.

4. Utilizar aplicaciones para realizar documentos en línea (ej.: google docs). 
JOSÉ MANUEL SÁNCHEZ SORDO

INTERVENCIÓN EN LÍNEA PARA EL AUMENTO DE LA AUTOEFICACIA EN HABILIDADES DOCENTES POR INTERNET ANTE LA CONTINGENCIA DEL COVID-19

5. Enseñar de manera asincrónica.

6. Desarrollar cursos digitales con una navegación simple e intuitiva.

7. Utilizar contenidos ya existentes en la web para mi docencia por internet.

8. Promover la autonomía y autorregulación del aprendizaje en línea de mis estudiantes.

9. Desarrollar actividades colaborativas en línea usando foros, wikis, blogs, etc.

10. Hacer uso óptimo de aulas virtuales y sus funcionalidades.

11. Supervisar a distancia actividades de práctica (experimentos, análisis de casos, recopilación de datos, etc.).

12. Planificar un horario para mis actividades docentes en línea (horario para responder correos con dudas, calificar actividades, reuniones en chat, etc.)

13. Utilizar tecnologías para desarrollar productos de aprendizaje como infografías, videos, animaciones, simuladores, etc.

14. Enseñar los contenidos de mi disciplina completamente en línea aun cuando éstos sean de índole aplicada.

15. Guiar al alumno en su búsqueda y generación de conocimiento en línea.

16. Participar en foros de discusión de manera constante como parte de mis actividades docentes.

17. Generar exámenes o cuestionarios en línea calificables de manera automática.

18. Evaluar de manera personalizada el aprendizaje de cada uno de mis estudiantes con base en las actividades desarrolladas en línea.

Cabe mencionar que con la primera aplicación de la escala (pretest) se incluyeron adicionalmente preguntas relacionadas con el interés de los participantes por tomar el curso y su opinión con relación a la educación a distancia en tiempos de COVID-19. De igual modo en la segunda aplicación de la escala (postest) se incluyeron preguntas sobre la utilidad del curso para sus futuras actividades docentes. Ninguna de estas preguntas afectaba la puntuación obtenida dentro de la escala de autoeficacia.

REACTIVOS ADICIONALES EN EL PRETEST:

1. Ante la emergencia sanitaria del Covid-19, aumentó mi interés en capacitarme sobre enseñanza por internet.

2. Ante situaciones de emergencia como la actual, considero como buena alternativa la enseñanza por internet para llevar a cabo mis labores docentes. Decidí tomar este curso principalmente debido a que:

a) Desconozco los principios básicos de la enseñanza por internet.

b) Ya tengo algunos conocimientos básicos sobre enseñanza por internet, pero quiero ampliarlos.

c) Mis conocimientos sobre enseñanza por internet son amplios, y el curso llamó mi atención. 
INTERVENCIÓN EN LÍNEA PARA EL AUMENTO DE LA AUTOEFICACIA EN HABILIDADES DOCENTES POR INTERNET ANTE LA CONTINGENCIA DEL COVID-19

REACTIVOS ADICIONALES EN EL POSTEST:

1. Este curso me sirvió para mejorar mis habilidades docentes en internet.

2. Con este curso amplié mis conocimientos sobre enseñanza y aprendizaje en línea.

3. Aplicaré algunas de las técnicas y metodologías revisadas a lo largo del curso a mis labores docentes.

Finalmente, una vez construida la arquitectura digital que alojó el curso, así como la escala de autoeficacia para la docencia por internet, se lanzó el 5 de abril una convocatoria en línea para docentes de la UNAM de nivel superior (universitario) y medio superior (bachillerato) que desearan inscribirse y participar. Dando inicio al curso el día 15 de abril y concluyéndolo el día 27 del mismo mes, cabe mencionar que la UNAM, debido a la contingencia, suspendió actividades presenciales el día 23 de marzo del 2020.

\section{RESULTADOS}

Como se ha mencionado en el apartado anterior, durante la primera aplicación de la escala se incluyeron reactivos adicionales en torno a conocer la experiencia con el uso de TiC de los docentes, así como sus opiniones y motivos para tomar el curso durante la contingencia del Covid-19, siendo los resultados de tales preguntas los siguientes:

GRÁFICA 1

Valoración de la experiencia con TIC

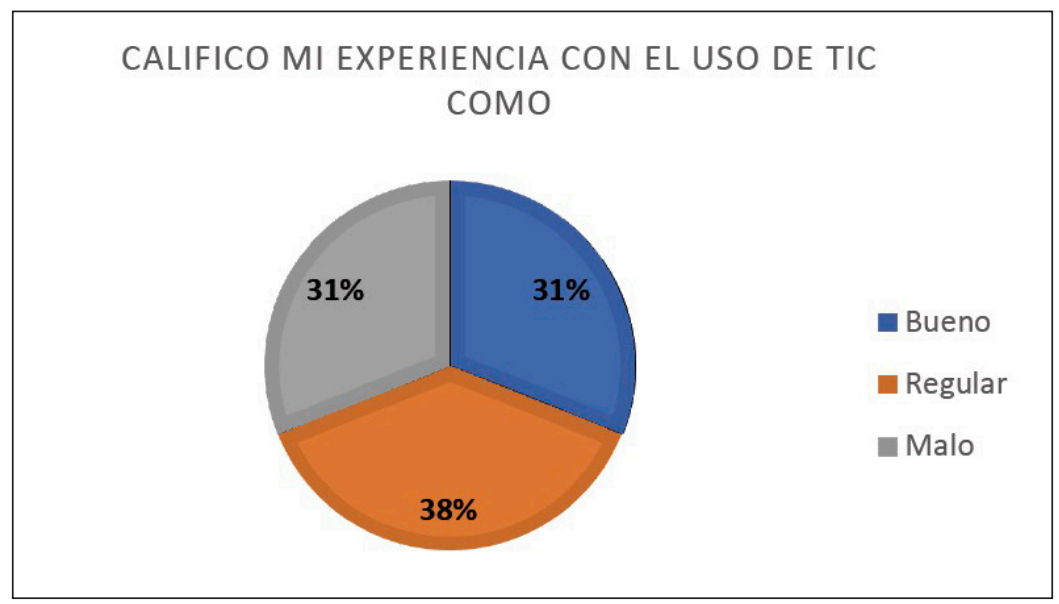



POR INTERNET ANTE LA CONTINGENCIA DEL COVID-19

GRÁFICA 2

Conocimiento sobre el uso de las TIC

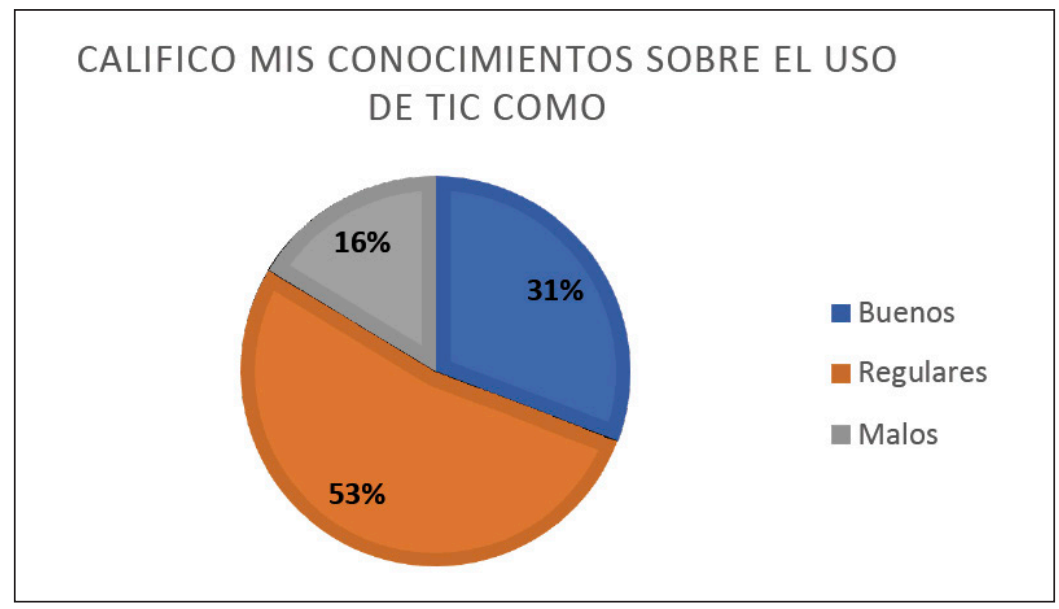

GRÁFICA 3

Interés en la capacitación en TIC

\section{ANTE LA EMERGENCIA SANITARIA DE LA COVID-19, AUMENTÓ MI INTERÉS EN CAPACITARME SOBRE ENSEÑANZA POR INTERNET}

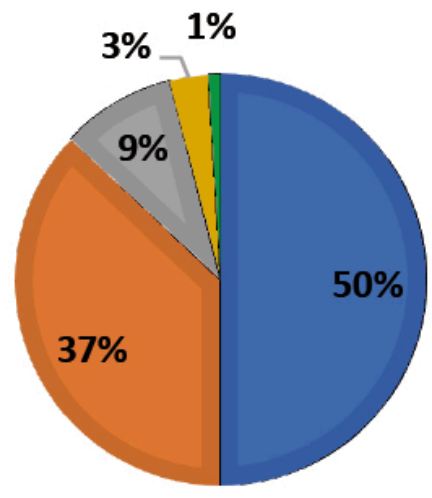

- Totalmente de acuerdo

De acuerdo

Ni de acuerdo ni en desaecuerdo

En desacuerdo

Totalmente en desacuerdo 
JOSÉ MANUEL SÁNCHEZ SORDO

INTERVENCIÓN EN LÍNEA PARA EL AUMENTO DE LA AUTOEFICACIA EN HABILIDADES DOCENTES POR INTERNET ANTE LA CONTINGENCIA DEL COVID-19

GRÁFICA 4

Enseñanza con TIC ante CoviD-19

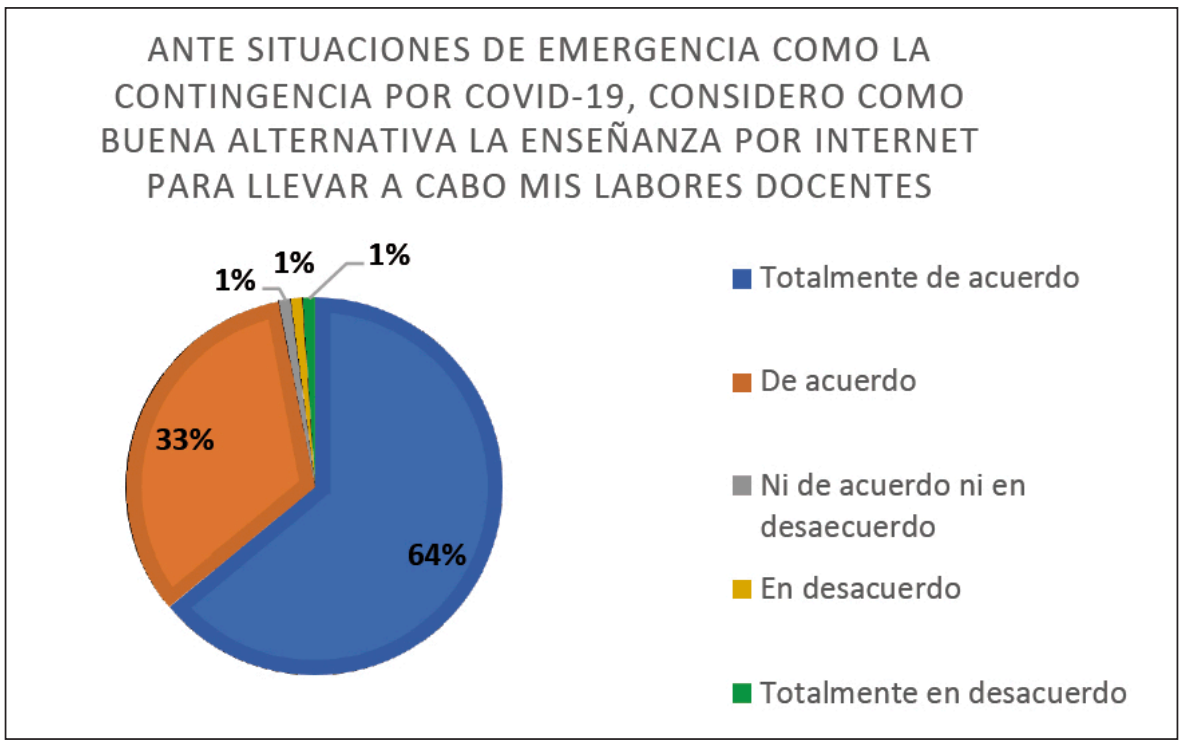

GRÁFICA 5

Realización de cursos

DECIDÍ TOMAR ESTE CURSO PRINCIPALMENTE DEBIDO A QUE

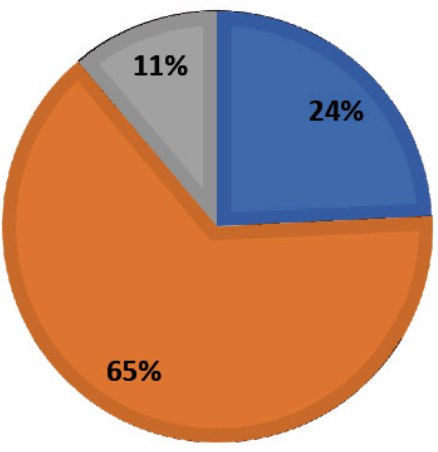

Desconocía los principios básicos de la enseñanza por internet.

Ya tenía algunos conocimientos básicos sobre enseñanza por internet pero quería ampliarlos.

- Mis conocimientos sobre enseñanza por internet son amplios, y el curso llamó mi atención. 
INTERVENCIÓN EN LÍNEA PARA EL AUMENTO DE LA AUTOEFICACIA EN HABILIDADES DOCENTES POR INTERNET ANTE LA CONTINGENCIA DEL COVID-19

RESULTADOS DE LA APLICACIÓN DE LA ESCALA DE AUTOEFICACIA PARA LA DOCENCIA POR INTERNET:

Una vez descargadas las bases de datos de las escalas aplicadas a los 70 participantes que acreditaron el curso se analizaron los datos haciendo uso del sPss versión 22. Al no obtener una distribución normal en los datos (Tabla 1), se utilizó estadística no paramétrica para la comparación de los grupos, particularmente la prueba de los rangos con signos de Wilcoxon, como se muestra más adelante.

TABLA 1

Prueba de Kolmogorov-Smirnov

\begin{tabular}{|l|l|c|c|}
\hline \multicolumn{2}{|l|}{} & PRETEST & POSTEST \\
\hline \multirow{2}{|l|}{$\mathrm{N}$} & Media & 70 & 70 \\
\cline { 2 - 4 } & DE & 110.21 & 130.46 \\
\hline \multirow{2}{*}{ Máxímas diferencias extremas } & Absoluta & 40.759 & 28.703 \\
\cline { 2 - 4 } & Positivo & .160 & .194 \\
\cline { 2 - 4 } & Negativo & .106 & .136 \\
\hline Estadístico de prueba & -.160 & -.194 \\
\hline Sig. asintótica (bilateral) & .160 & .194 \\
\hline
\end{tabular}

En la Tabla 1, se aprecia que los niveles obtenidos para la prueba de normalidad son significativos $(p<.05)$, lo cual indica que los datos obtenidos en la muestra no siguen la normalidad.

TABLA 2

Estadísticos de puntuaciones obtenidas (pretest)

\begin{tabular}{|c|c|c|c|c|c|}
\hline PREGUNTA & $\mathrm{N}$ & MÍNIMO & MÁXIMO & MEDIA & $\mathrm{S}$ \\
\hline Ítem 3 & $\mathbf{7 0}$ & $\mathbf{. 0 0}$ & $\mathbf{9 . 0 0}$ & $\mathbf{5 . 6 0 0 0}$ & $\mathbf{2 . 9 8 9 8 4}$ \\
\hline Ítem 9 & $\mathbf{7 0}$ & $\mathbf{. 0 0}$ & $\mathbf{9 . 0 0}$ & $\mathbf{5 . 6 5 7 1}$ & $\mathbf{2 . 9 4 8 2 8}$ \\
\hline Ítem 6 & 70 & .00 & 9.00 & 5.7286 & 2.94845 \\
\hline Ítem 14 & 70 & .00 & 9.00 & 5.7571 & 2.69425 \\
\hline Ítem 16 & 70 & .00 & 9.00 & 5.7857 & 2.86858 \\
\hline Ítem 13 & 70 & .00 & 9.00 & 5.8571 & 2.78342 \\
\hline
\end{tabular}


JOSÉ MANUEL SÁNCHEZ SORDO

INTERVENCIÓN EN LÍNEA PARA EL AUMENTO DE LA AUTOEFICACIA EN HABILIDADES DOCENTES POR INTERNET ANTE LA CONTINGENCIA DEL COVID-19

\begin{tabular}{|c|c|c|c|c|c|}
\hline PREGUNTA & $\mathrm{N}$ & MÍNIMO & MÁXIMO & MEDIA & $\mathrm{S}$ \\
\hline Ítem 1 & 70 & .00 & 9.00 & 5.9000 & 2.53183 \\
\hline Ítem 11 & 70 & .00 & 9.00 & 5.9143 & 2.60847 \\
\hline Ítem 10 & 70 & .00 & 9.00 & 5.9286 & 2.65025 \\
\hline Ítem 17 & 70 & .00 & 9.00 & 5.9857 & 3.09039 \\
\hline Ítem 5 & 70 & .00 & 9.00 & 6.2286 & 2.61640 \\
\hline Ítem 8 & 70 & .00 & 9.00 & 6.3143 & 2.24282 \\
\hline Ítem 18 & 70 & .00 & 9.00 & 6.3286 & 2.82700 \\
\hline Ítem 2 & 70 & .00 & 9.00 & 6.3429 & 2.51883 \\
\hline Ítem 15 & 70 & .00 & 9.00 & 6.4571 & 2.19806 \\
\hline Ítem 12 & 70 & 1.00 & 9.00 & 6.6286 & 2.28525 \\
\hline Ítem 7 & 70 & .00 & 9.00 & 6.8000 & 2.08236 \\
\hline Ítem 4 & 70 & .00 & 9.00 & 7.0000 & 2.42571 \\
\hline
\end{tabular}

Como puede verse en la Tabla 2, el promedio de la media de respuesta de los ítems es de 6.12, siendo los reactivos con puntuaciones más bajas el 3 (Sostener videoconferencias dinámicas con mis estudiantes que no excedan los 45 minutos) y el 9 (Desarrollar actividades colaborativas en línea usando foros, wikis, blogs, etc.).

TABLA 3

Estadísticos de puntuaciones obtenidas (postest)

\begin{tabular}{|c|c|c|c|c|c|}
\hline PREGUNTA & $\mathrm{N}$ & MíNIMO & MÁXIMO & MEDIA & $\mathrm{S}$ \\
\hline Ítem 3 & 70 & .00 & 9.00 & 6.8143 & 2.33619 \\
\hline Ítem 6 & 70 & 1.00 & 9.00 & 6.8857 & 2.03977 \\
\hline Ítem 9 & 70 & 1.00 & 9.00 & 6.9286 & 1.99508 \\
\hline Ítem 14 & 70 & .00 & 9.00 & 6.9714 & 1.98525 \\
\hline Ítem 17 & $\mathbf{7 0}$ & $\mathbf{. 0 0}$ & $\mathbf{9 . 0 0}$ & $\mathbf{7 . 0 0 0 0}$ & $\mathbf{2 . 2 5 8 6 4}$ \\
\hline Ítem 16 & 70 & .00 & 9.00 & 7.0000 & 2.00723 \\
\hline Ítem 1 & 70 & .00 & 9.00 & 7.0143 & 1.86106 \\
\hline Ítem 11 & 70 & 1.00 & 9.00 & 7.0857 & 1.96891 \\
\hline
\end{tabular}


JOSÉ MANUEL SÁNCHEZ SORDO

INTERVENCIÓN EN LÍNEA PARA EL AUMENTO DE LA AUTOEFICACIA EN HABILIDADES DOCENTES POR INTERNET ANTE LA CONTINGENCIA DEL COVID-19

\begin{tabular}{|c|c|c|c|c|c|}
\hline PREGUNTA & $\mathrm{N}$ & MíNIMO & MÁXIMO & MEDIA & $\mathrm{S}$ \\
\hline Ítem 10 & 70 & 1.00 & 9.00 & 7.1286 & 1.84093 \\
\hline Ítem 12 & 70 & 1.00 & 9.00 & 7.3000 & 1.83643 \\
\hline Ítem 18 & $\mathbf{7 0}$ & $\mathbf{2 . 0 0}$ & $\mathbf{9 . 0 0}$ & $\mathbf{7 . 3 1 4 3}$ & $\mathbf{2 . 0 2 5 5 1}$ \\
\hline Ítem 13 & 70 & 1.00 & 9.00 & 7.3571 & 1.88076 \\
\hline Ítem 2 & 70 & 2.00 & 9.00 & 7.5000 & 1.60389 \\
\hline Ítem 5 & 70 & 2.00 & 9.00 & 7.5286 & 1.89371 \\
\hline Ítem 8 & 70 & 1.00 & 9.00 & 7.5857 & 1.49872 \\
\hline Ítem 15 & 70 & 1.00 & 9.00 & 7.5857 & 1.68963 \\
\hline Ítem 4 & 70 & 1.00 & 9.00 & 7.6286 & 1.88949 \\
\hline Ítem 7 & 70 & 4.00 & 9.00 & 7.8286 & 1.39327 \\
\hline
\end{tabular}

En la Tabla 3, se aprecia que la media de respuesta de los participantes aumentó durante el postest (7.24). Los reactivos en los cuales hubo mayor aumento en sus puntuaciones medias en comparación con la primera aplicación de la escala son «17. Generar exámenes o cuestionarios en línea calificables de manera automática» y «18. Evaluar de manera personalizada el aprendizaje de cada uno de mis estudiantes con base en las actividades desarrolladas en línea». Mas los reactivos que aún presentan las puntuaciones más bajas son «3. Sostener videoconferencias dinámicas con mis estudiantes que no excedan los 45 minutos» y «6. Desarrollar cursos digitales con una navegación simple e intuitiva». Lo cual indica que ambas situaciones son problemáticas para los docentes.

TABLA 4

Estadísticos descriptivos de las aplicaciones pretest-postest

\begin{tabular}{|c|c|c|c|c|c|c|c|c|}
\hline Puntuaciones & $\mathrm{N}$ & Media & $\mathrm{S}$ & Mínimo & Máximo & $\begin{array}{c}\text { PercentiL } \\
25\end{array}$ & $\begin{array}{c}\text { PercentIL } \\
50\end{array}$ & $75^{\circ}$ \\
\hline Antes & 70 & 110.21 & 40.759 & 10 & 162 & 79.00 & 123.00 & 143.00 \\
\hline Después & 70 & 130.46 & 28.703 & 40 & 162 & 121.75 & 140.00 & 150.00 \\
\hline
\end{tabular}

Como puede observase en la Tabla 4, la media global de puntuaciones de la escala en el pretest era de 110, mientras que al postest fue de 130, de igual modo la mediana (percentil 50) se encuentra en la puntuación 123 para la aplicación anterior al curso, mientras que la mediana se ubica en la puntuación 140 para las puntuaciones obtenidas después del curso. Lo cual indica un aumento significativo 
INTERVENCIÓN EN LÍNEA PARA EL AUMENTO DE LA AUTOEFICACIA EN HABILIDADES DOCENTES POR INTERNET ANTE LA CONTINGENCIA DEL COVID-19

en las puntuaciones de autoeficacia de los participantes que tomaron el curso como se muestra a continuación.

TABLA 5

Prueba de Wilcoxon de los rangos con signo

\begin{tabular}{|l|l|c|r|r|}
\hline \multicolumn{2}{|c|}{} & N & \multicolumn{1}{|c|}{$\begin{array}{c}\text { RANGO } \\
\text { PROMEDIO }\end{array}$} & $\begin{array}{c}\text { SUMA DE } \\
\text { RANGOS }\end{array}$ \\
\hline \multirow{3}{*}{ Pretest-Postest } & Rangos negativos & 13 & 23.69 & 308.00 \\
\cline { 2 - 5 } & Rangos positivos & 55 & 37.05 & 2038.00 \\
\cline { 2 - 5 } & Empates & 2 & & \\
\cline { 2 - 5 } & Total & 70 & & \\
\hline
\end{tabular}

En la Tabla 5, se muestra cómo 13 de los 70 docentes evaluados disminuyeron en sus puntuaciones de autoeficacia después del curso, lo cual indica que en esos casos el curso no ayudó a aumentar la autoeficacia. Mas por el contrario podemos observar que la mayoría (55) de los evaluados sí aumentó sus puntuaciones al término del curso (postest) en comparación con la evaluación inicial (pretest), de igual modo hubo dos casos que se mantuvieron iguales (empates) en ambas mediciones.

TABLA 6

Estadísticos de prueba

\begin{tabular}{|c|c|}
\hline & Pretest-postest \\
\hline$Z$ & -5.286 \\
\hline Sig. asintótica (bilateral) & $\mathbf{. 0 0 0}$ \\
\hline
\end{tabular}

En la Tabla 6, se aprecia que sí hay diferencias significativas en las puntuaciones de autoeficacia antes y después de haber tomado el curso con un intervalo de confianza del $95 \%$, dado que los valores de Z (-5.28) son significativos a .000 para la prueba de Wilcoxon de los rangos con signo. Lo cual permite rechazar la hipótesis nula. 

POR INTERNET ANTE LA CONTINGENCIA DEL COVID-19

GRÁFICA 6

Rangos de disminución/aumento después del curso

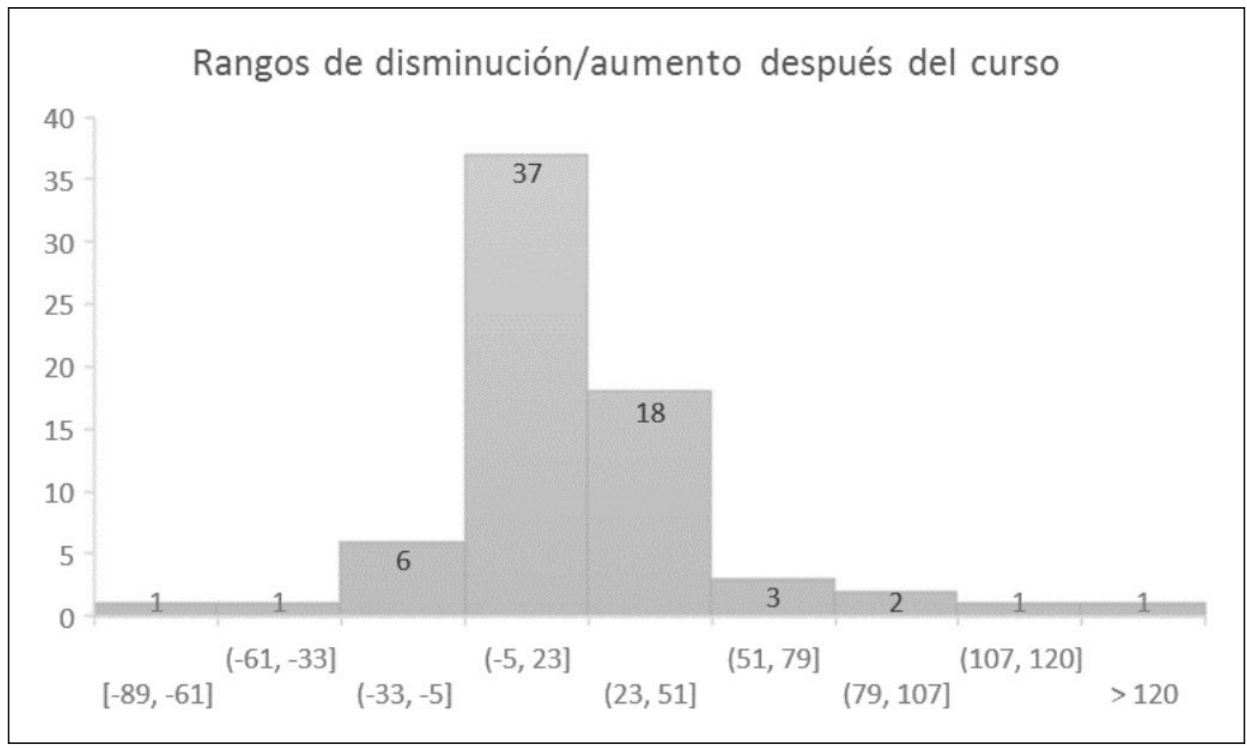

Como se muestra en la Gráfica 6, la mayor parte de los participantes (45) se concentra en los rangos que van de -5 a 51 puntos en cuanto a la disminución o aumento en las puntuaciones de la escala de autoeficacia después de haber tomado el curso. Lo cual indica un incremento positivo en las creencias de la mayoría de los evaluados, situación que se muestra detalladamente en la siguiente gráfica.

Como se observa en la Gráfica 7, la mayoría de los sujetos (55) aumentaron sus puntuaciones después del curso (diferencia), mientras que 13 disminuyeron, lo cual indica que algunos de los participantes evaluados no mejoraron sus creencias de autoeficacia después de haber tomado el curso, como es el caso del sujeto 6, cuyas puntuaciones en la escala disminuyeron 89 puntos en la segunda aplicación de la escala (pretest: 151 y postest: 65). Caso opuesto es el del sujeto 13, cuyas puntuaciones iniciales fueron de 10 mientras que las finales de 153, lo cual indica un aumento de 143 puntos. En promedio los 55 participantes que aumentaron sus puntuaciones lo hicieron 28.9 puntos y en promedio los 13 participantes que disminuyeron sus puntuaciones lo hicieron en -17.9 puntos en la escala de autoeficacia. Con lo cual podemos inferir que la mayoría de los participantes se vieron beneficiados por tomar el curso «Enseñanza Asistida por Computadora en la Era Digital", ya que sus creencias sobre sus propias capacidades en torno al desarrollo de actividades docentes en línea aumentaron significativamente al término de este. 
INTERVENCIÓN EN LÍNEA PARA EL AUMENTO DE LA AUTOEFICACIA EN HABILIDADES DOCENTES POR INTERNET ANTE LA CONTINGENCIA DEL COVID-19

GRÁFICA 7

Puntuaciones pretest-postest y línea de aumento o disminución por participante

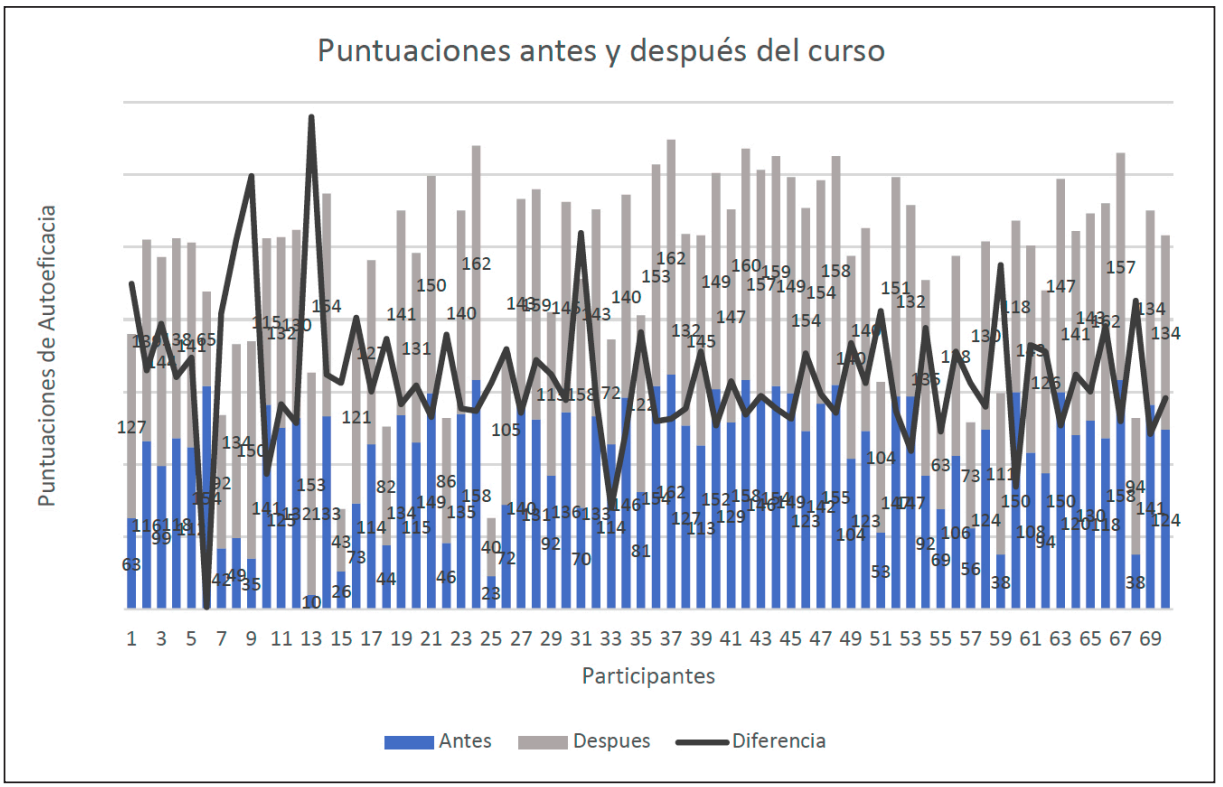

REACTIVOS ADICIONALES EN EL POSTEST:

GRÁFICA 8

Frecuencia de participantes para la afirmación «Este curso me sirvió para mejorar mis habilidades docentes en internet»

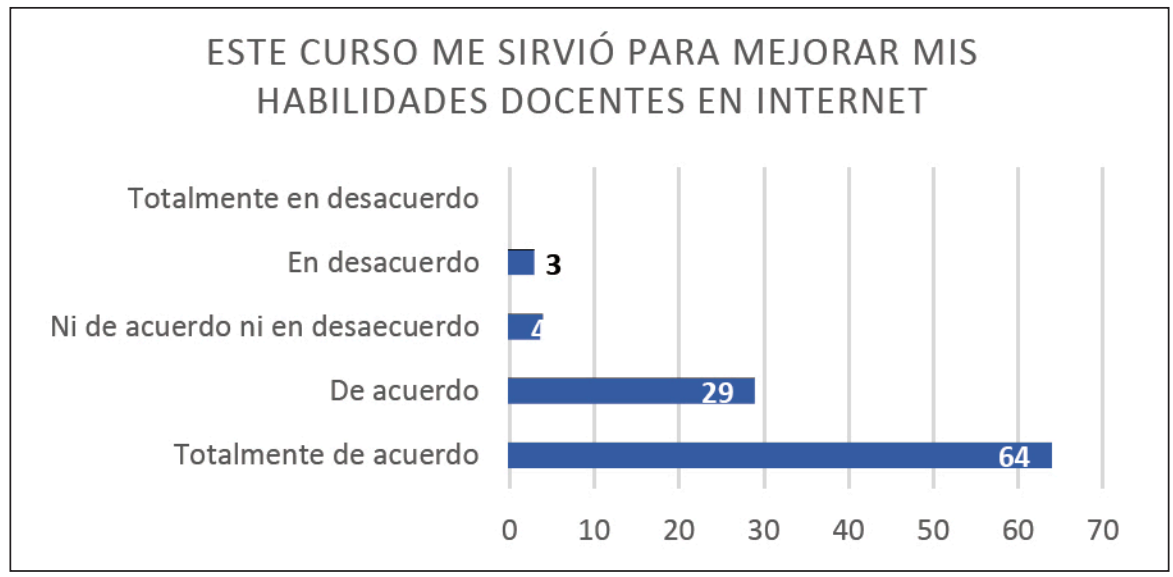


INTERVENCIÓN EN LÍNEA PARA EL AUMENTO DE LA AUTOEFICACIA EN HABILIDADES DOCENTES POR INTERNET ANTE LA CONTINGENCIA DEL COVID-19

En la Gráfica 8, se muestra que, de los 70 participantes, 45 reportan estar totalmente de acuerdo con que el curso sirvió para mejorar sus habilidades docentes en internet. Lo cual, por un lado, ratifica el fin último del curso como herramienta de capacitación docente y, por el otro, podría sugerirse que si el curso les ayudó a mejorar sus habilidades docentes en internet esto impactó en el aumento significativo de sus creencias de autoeficacia (Tablas 3 y 5).

GRÁFICA 9

Frecuencia de participantes para la afirmación «Con este curso amplié mis conocimientos sobre enseñanza y aprendizaje en línea»

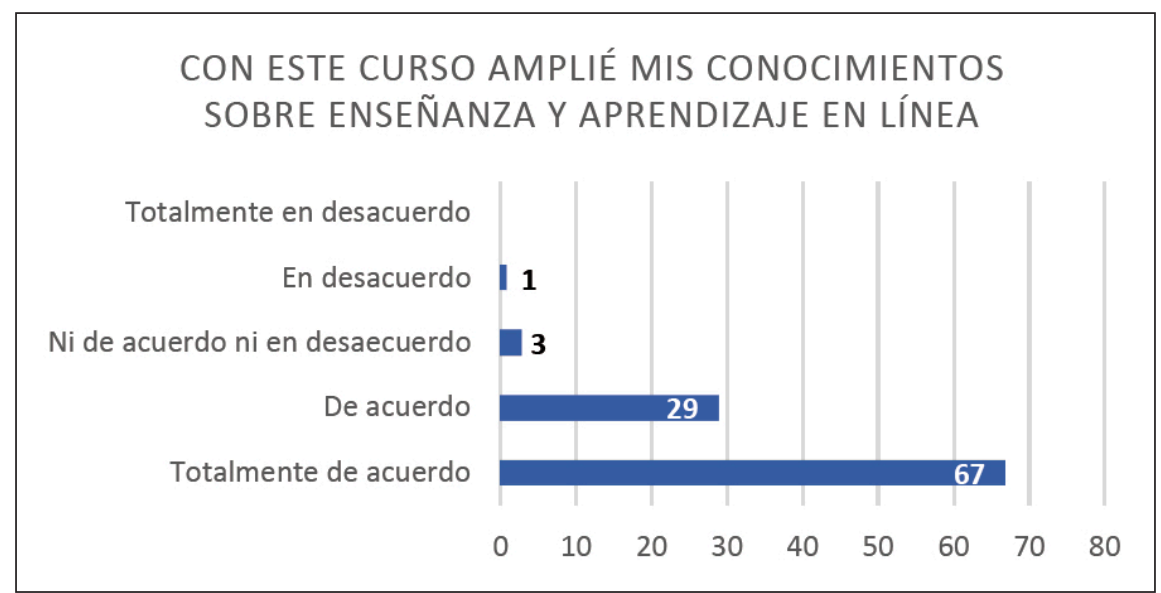

En la Gráfica 9, la mayor parte de los participantes (47) reporta estar totalmente de acuerdo de que con el curso ampliaron sus conocimientos sobre enseñanza y aprendizaje en línea, lo cual pudo haber fungido como fuente de autoeficacia para el aumento en sus creencias.

Finalmente, cabe mencionar que la mayoría de los participantes (50) reporta según la Gráfica 10 estar totalmente de acuerdo en aplicar algunas de las técnicas y metodologías revisadas a lo largo del curso en sus labores docentes por internet.

\section{DisCUSIÓN Y CONCLUSIONES}

Con base en los resultados obtenidos, podemos comentar que los participantes que se han visto obligados a digitalizar sus labores presenciales de docencia consideran el uso de las tecnologías de la información y comunicación como medios idóneos para llevar a cabo sus tareas de enseñanza, mas la mayoría de ellos carecía de formación formal al respecto. Por lo cual, ante la situación emergente del coviD19 su interés en recibir capacitación sobre la modalidad educativa a distancia y por internet aumentó considerablemente, lo cual habla de una imperante necesidad 
INTERVENCIÓN EN LÍNEA PARA EL AUMENTO DE LA AUTOEFICACIA EN HABILIDADES DOCENTES POR INTERNET ANTE LA CONTINGENCIA DEL COVID-19

\section{GRÁFICA 10}

Frecuencia de participantes para la afirmación "Aplicaré algunas de las técnicas y metodologías revisadas a lo largo del curso a mis labores docentes»

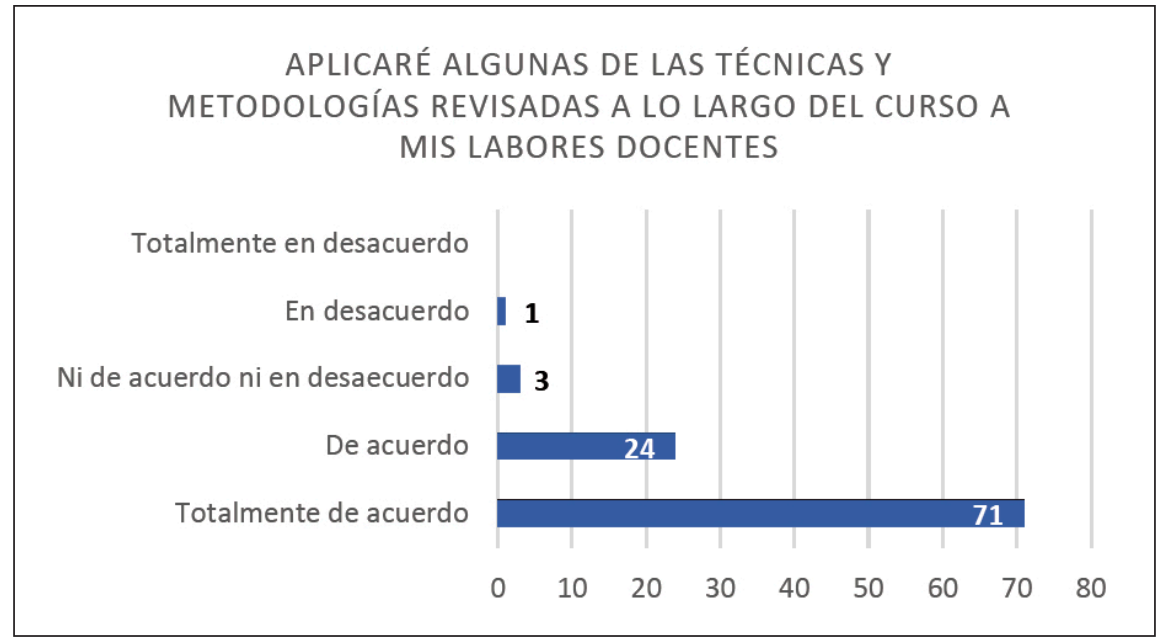

de alfabetización digital con enfoque estrictamente pedagógico por parte de las instituciones educativas mexicanas, que deberá centrarse principalmente en el uso educativo de la tecnología a la vez que ilustre su aspecto utilitario, pues la brecha digital en el contexto educativo mexicano es amplia y puede ser mitigada por medio de la instrucción en competencias digitales para los docentes y estudiantes con intervenciones similares a la aquí expuesta, que aborden teorías, metodología y técnicas de enseñanza y aprendizaje en contextos digitales.

A nivel psicológico uno de los principales componentes que influyen en la conducta son las creencias que las personas tenemos sobre nuestra propia capacidad para hacer bien o mal determinadas acciones, y como puede verse en los resultados de esta investigación dichas creencias de autoeficacia pueden ser modificadas con intervenciones sencillas que brinden al participante conocimientos y habilidades conductuales que le hagan ser y creerse más eficaz para llevar a cabo sus actividades.

En el caso concreto de este estudio se encontró que un pequeño porcentaje (18\%) de la muestra disminuyó sus niveles de autoeficacia después de haber tomado el curso, lo cual posiblemente infiere que los contenidos abordados o las actividades indicadas en el curso fueron percibidas como muy complejas, siendo entonces necesario realizar intervenciones de formación y capacitación más sencillas para aquellos docentes que recién incursionan en la tecnología con fines educativos; mas también debe recalcarse que la mayoría de los participantes ( $81 \%$ ) consiguió un aumento significativo en sus niveles de autoeficacia para realizar actividades docentes por internet después de haber acreditado el curso. De igual modo 
JOSÉ MANUEL SÁNCHEZ SORDO

INTERVENCIÓN EN LÍNEA PARA EL AUMENTO DE LA AUTOEFICACIA EN HABILIDADES DOCENTES POR INTERNET ANTE LA CONTINGENCIA DEL COVID-19

es importante mencionar que los participantes, a la par del curso ofertado en esta investigación, continuaron impartiendo sus clases por internet, lo cual pudo influir en el aumento o disminución de la autoeficacia evaluada al término del curso, sin embargo, dicha situación no resta importancia al impacto de la intervención aquí desarrollada.

\section{REFERENCIAS BIBLIOGRÁFICAS}

Adell Segura, J. y Sales Ciges, A. (1999). El profesor online: elementos para la definición de un nuevo rol docente. En EDUTEC 99. IV Congreso de Nuevas Tecnologías de la Información y Comunicación para la educación. Nuevas tecnologías en la formación flexible y a distancia. (1999). Universidad de Sevilla. Secretariado de Recursos Audiovisuales y Nuevas Tecnologías.

Amador, R. (2020). Aprende en casa con \#SanaDistancia en tiempos de \#COVID-19. En. IISUE. Educación y pandemia. Una visión académica. México: UNAM.

Area, M. y Adell, J. (2009): eLearning: Enseñar y aprender en espacios virtuales. En J. De Pablos (Coord.). Tecnología Educativa. La formación del profesorado en la era de Internet (pp. 391-424). Málaga: Aljibe.

Area, M. \& Adell, J. (2009). E-learning: enseñar y aprender en espacios virtuales. Tecnología Educativa. La formación del profesorado en la era de Internet, 391-424.

Arrieta, A. y Montes, D. (2011). Alfabetización digital: El uso de las TiC’s más allá de una formación instrumental y una buena infraestructura. Revista Colombiana de Ciencia Animal, 1(3), 180-197.

Bandura, A. (1997). Self-efficacy: The exercise of Control. New York: Freeman.

Bandura, A. (2006). Guide to constructing self-efficacy scales. En F. Pajares y T. Urdan (Eds.), Self-efficacy beliefs of adolescents (pp. 307-337). Greenwich, ст: Information Age.

Bandura, A. (2010). Auto-Eficacia: "Cómo afrontamos los cambios de la Sociedad actual». Bilbao: Descleé De Brouwer.

Casanova, H. (2020). Presentación. En IISUE. Educación y pandemia. Una visión académica. México: UNAM.

Díaz, M. (2020), Equipos directivos de educación primaria. Improvisar la alfabetización digital durante la cuarentena. En IISUE. Educación y pandemia. Una visión académica. México: UNAM.

Downes S. (2012). Connectivism and Connective Knowledge. UPAEP.

Guimarães, I.; Tejada, J. y Pozos, K. (2019), Formación docente para educación a distancia: la construcción de competencias digitales. Universidade Católica Dom Bosco, Brasil, 24(51).

Lawshe, C. (1975). A quantitive approach to content validity. Personnel Psychology, 28, 563-575.

Lloyd, M. (2020). Desigualdades educativas y brecha digital en tiempos de COVID-19. En IISUE. Educación y pandemia. Una visión académica. México: UNAM.

López, M. y Chávez, J. (2013). La formación de profesores universitarios en aplicación de las Tic. Sinéctica, 41. http://www.sinectica.iteso.mx/articulo/?id=41_la_formacion_deprofesores_universitarios_en_la_aplicacion_de_las_tic.

Maya, A. (2019). Orientaciones básicas sobre educación a distancia y la función tutorial. UNESCO - San José, Costa Rica. 
INTERVENCIÓN EN LÍNEA PARA EL AUMENTO DE LA AUTOEFICACIA EN HABILIDADES DOCENTES POR INTERNET ANTE LA CONTINGENCIA DEL COVID-19

Moreno, G. y Gonzáles, D. (2013). Conocimiento y uso de competencias docentes sobre las tic en educación superior. EDUTEC.

Pons, A. (2013). El desorden digital guía para historiadores y humanistas. Madrid, España: Siglo XXI.

Ruiz, E. (2020). La práctica docente universitaria en ambientes de educación a distancia. Tensiones y experiencias cambio. En IISUE. Educación y pandemia. Una visión académica. México: UnAM.

Sánchez, J. (2019a). Data mining techniques for the study of online learning from an extended approach. Multidisciplinary Journal for Education, Social and Technological Sciences, 6(1), 1-24.

Sánchez, J. (2019b). Desarrollo de un entorno digital de aprendizaje desde el Conectivismo y su posterior análisis utilizando algoritmos de machine learning. Edutec. Revista Electrónica de Tecnología Educativa, 69, 1-22. https://doi.org/10.21556/edutec.2019.69.1355.

Siemens, G. (2006). Conociendo el conocimiento. Madrid: Ediciones Nodos Ele.

Silva, A. (2004). Métodos cuantitativos en Psicología. México: Trillas.

Suárez, J. y Anaya, D. (2004). Educación a distancia y presencial: Diferencias en componentes cognitivo y motivacional de estudiantes universitarios. Revista Iberoamericana de Educación a Distancia, 7(1/2), 65-75.

Tristán, A. (2008). Modificación al modelo de Lawshe para el dictamen cuantitativo de la validez de contenido en un instrumento objetivo. Avances en Medición, 6, 37-48.

UNESCO (2020, 3 de junio). Cómo planificar las soluciones de aprendizaje a distancia durante el cierre temporal de las escuelas. https://es.unesco.org/news/como-planificarsoluciones-aprendizaje-distancia-durante-cierre-temporal-escuelas.

Vanden, A. (2020, 11 de marzo). 10 Tips for First-Time Online Faculty. https://medium. com/@andrewvandenheuvel/10-tips-for-first-time-online-professors-6373ca1c5c40. 
